\title{
Comparison of circulating tumor cell (CTC) detection rates with epithelial cell adhesion molecule (EpCAM) and cell surface vimentin (CSV) antibodies in different solid tumors: a retrospective study
}

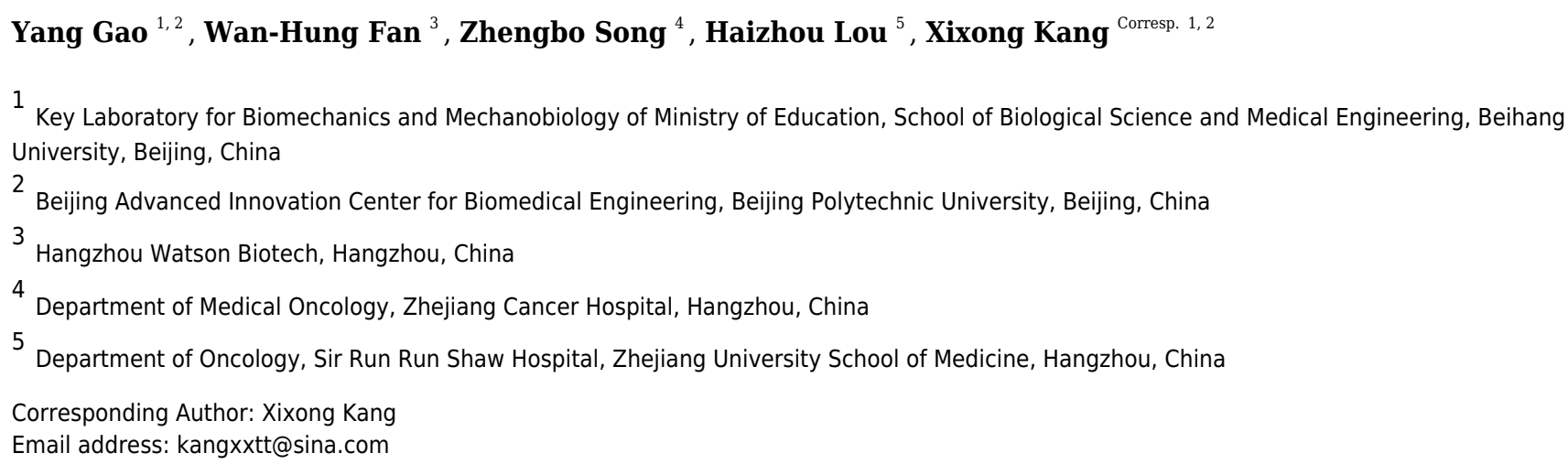

Purpose: Status of epithelial-mesenchymal transition (EMT) varies from tumors to tumors. Epithelial cell adhesion molecule (EpCAM) and cell surface vimentin (CSV) are the most common used targets for isolating epithelial and mesenchymal CTCS, respectively. This study aimed to identify a suitable CTC capturing antibody for CTC enrichment in each solid tumor by comparing CTC detection rates with EpCAM and CSV antibodies in different solid tumors.Methods: Treatment-naive patients with confirmed cancer diagnosis and healthy people who have performed CTC detection between April 2017 and May 2018 were included in this study. CTC detection was performed with CytoSorter ${ }^{\circledR}$ CTC system using either EpCAM or CSV antibody. In total, 853 CTC results from 690 cancer patients and 72 healthy people were collected for analysis. The performance of CTC capturing antibody was determined by the CTC detection rate.Results: EPCAM has the highest CTC detection rate of $84.09 \%$ in CRC, followed by BCa (78.32\%). CTC detection rates with EpCAM antibody are less than $40 \%$ in HCC (25\%), PDAC (32.5\%) and OC (33.33\%). CSV has the highest CTC detection rate of $90 \%$ in sarcoma, followed by BC (85.71\%), UC (84.62\%), OC $(83.33 \%)$ and $\mathrm{BCa}(81.82 \%)$. CTC detection rates with CSV antibody are over $60 \%$ in all 14 solid tumors. Except for CRC, CSV has better performances than EpCAM in most solid tumors regarding the CTC detection rates.Conclusion: EpCAM can be used as a target to isolate CTCs in CRC, LC, GC, BCa, EC, HNSCC, CC and PCa, especially in CRC, while CSV can be used in most solid tumors for isolating CTCs. 
1 Original Article

2 Comparison of circulating tumor cell (CTC) detection rates with epithelial cell

3 adhesion molecule (EpCAM) and cell surface vimentin (CSV) antibodies in

4 different solid tumors: a retrospective study

5 Yang GAO ${ }^{1,2}$, Wan-Hung FAN ${ }^{3}$, Zhengbo $\mathrm{SONG}^{4}$, Haizhou $\mathrm{LOU}^{5}$, Xixong $\mathrm{KANG}^{1,2}$

$8 \quad{ }^{1}$ Key Laboratory for Biomechanics and Mechanobiology of Ministry of Education, School of Biological Science 9 and Medical Engineering, Beihang University, Beijing, China

$10{ }^{2}$ Beijing Advanced Innovation Center for Biomedical Engineering, Beihang University, Beijing, China

$11{ }^{3}$ Hangzhou Watson Biotech. Inc, Hangzhou, Zhejiang, China

${ }^{4}$ Department of Medical Oncology, Zhejiang Cancer Hospital, Hangzhou, Zhejiang, China

${ }^{5}$ Department of Oncology, Sir Run Run Shaw Hospital, Zhejiang University School of Medicine, Hangzhou, Zhejiang, China and Medical Engineering, Beihang University, Beijing, China 
21 Tel: +86-10-59976611; Fax: +86-10-59976611

22 E-mail: kangxxtt@sina.com 
24

25

26

27

28

\section{Abstract}

Purpose: Status of epithelial-mesenchymal transition (EMT) varies from tumor to tumor. Epithelial cell adhesion molecule (EpCAM) and cell surface vimentin (CSV) are the most common used targets for isolating epithelial and mesenchymal CTCs, respectively. This study aimed to identify a suitable CTC capturing antibody in each solid tumor for CTC enrichment by comparing CTC detection rates with EpCAM and CSV antibodies in different solid tumors.

Methods: Treatment-naive patients with confirmed cancer diagnosis and healthy people who have performed CTC detection between April 2017 and May 2018 were included in this study. CTC detection was performed with CytoSorter ${ }^{\circledR}$ CTC system using either EpCAM or CSV antibody. In total, $853 \mathrm{CTC}$ results from 690 cancer patients and 72 healthy people were collected for analysis. The performance of CTC capturing antibody was determined by the CTC detection rate.

Results: EpCAM has the highest CTC detection rate of $84.09 \%$ in $\mathrm{CRC}$, followed by $\mathrm{BCa}$ (78.32\%). CTC detection rates with EpCAM antibody are less than 40\% in HCC (25\%), PDAC (32.5\%) and OC (33.33\%). CSV has the highest CTC detection rate of 90\% in sarcoma, followed by $\mathrm{BC}(85.71 \%)$, UC (84.62\%), OC (83.33\%) and $\mathrm{BCa}(81.82 \%)$. CTC detection rates with CSV antibody are over $60 \%$ in all 14 solid tumors. Except for CRC, CSV has better performances than EpCAM in most solid tumors regarding the CTC detection rates.

Conclusion: EpCAM can be used as a target to isolate CTCs in CRC, LC, GC, BCa, EC, HNSCC, CC and PCa, especially in CRC, while CSV can be used in most solid tumors for isolating CTCs. 


\section{Introduction}

Circulating tumor cells (CTCs) are tumor cells that have shed from tumor tissues and escaped into circulation [1]. CTCs are circulating within the bloodstream and can recolonize a distant site under a suitable condition, a phenomenon called metastasis, which is the main cause of death in

47 most cancer patients [1]. CTCs represent the undergoing process of metastasis and therefore

48 American Joint Committee on Cancer (AJCC) has introduced a new cancer stage, cM0 (i+), in the $497^{\text {th }}$ edition of Staging Manual for breast cancer $(\mathrm{BCa})[2]$. cM0 (i+) is defined as a tumor stage

50 when no clinical or radiographic evidence of distant metastases is found, but tumors cells are still

51 detected in the bone marrow, blood or distant non-regional lymph nodes [2]. The clinical values

52 of CTCs have been written in the Chinese expert consensus in lung cancer (LC), colorectal cancer

53 (CRC), BCa, esophageal cancer (EC), hepatocellular carcinoma (HCC) and pancreatic cancer [3-

54 8], and demonstrated in the prediction of patients' prognosis, monitoring of tumor recurrence, cancer diagnosis and screening, evaluation of the treatment responses, and guidance of treatment $[1]$.

It is estimated that $3.2 \times 10^{6}$ tumor cells detach from one gram of tumor tissue per day [9], but most of them quickly proceed to apoptosis due to the loss of adhesion to the extracellular matrix, hemodynamic shear forces, or attacks from the immune systems [1,9], which contributes

61 heterogeneity. Even CTCs from the same patients may differ in terms of cell size, morphology, 
63 CTCs, while discarding the majority of the surrounding blood cells. Thus, the first step of CTC 64 detection is to isolate CTCs from the background of blood cells, followed by an identification step

that can further distinguish (and possibly characterize) CTCs from the remaining cells. Many techniques have been developed to enrich CTCs, depending on either the unique biophysical or biochemical properties of CTCs [10]. Biophysical methods, also known as label-free methods, separate CTCs by size, density, electric charge, and deformability, while biochemical methods rely on the recognition of different surface biomarkers between CTCs and leukocytes. An ideal biomarker for CTCs isolation should be some surface molecules expressed universally and only on CTCs. Unfortunately, such molecule is not yet found. The most commonly used target for positive immuno-selection of CTCs is the epithelial cell adhesion molecule (EpCAM) [1], a cell surface glycoprotein typically over-expressed in epithelial cancer cells. EpCAM is not expressed on tumors of mesodermal and ectodermal origin, such as neurogenic tumors, sarcomas, melanomas, or lymphomas [11]. As the first and only US Food and Drug Administration (FDA) approved CTC detection technique, CellSearch ${ }^{\circledR}$ uses EpCAM antibody functionalized magnetic beads and cytokeration (CK) antibody to capture and identify CTCs, respectively [12]. A major drawback of EpCAM based methods is their inability to detect EpCAM negative CTCs. Epithelialmesenchymal transition (EMT) is a cellular process in which adherent epithelial cells acquire a migratory mesenchymal phenotype, and usually observed during morphogenesis at the time of embryonic development [13]. Studies have shown that EMT is quite common in tumor and plays an important role during metastasis, allowing tumor cells to become more aggressive, chemoresistant and invasive [13-15]. CTCs that have undergone EMT survive better in the circulation. 
84 EMT process is usually accompanied by the over-expression of mesenchymal markers, such as

85

86

87

88

89

90

91

92

93

94 vimentin, N-cadherin and Twist, while reducing expression of epithelial makers, such as EpCAM, cytokeratin (CK) and E-cadherin [16]. Thus, EMT induces the generation of EpCAM-negative CTCs. In fact, CTCs at different EMT states could be found in the circulation [17-18]. EpCAM antibody might not be able to capture the EpCAM-negative CTCs, which should be the majority of surviving CTCs in the circulation.. mesenchymal cells [19]. As EMT of cancer cells induces the over-expression of vimentin and translocation of vimentin to the cell surface [19], Satelli et al tried to raise an antibody against cell surface vimentin (CSV) to capture the EMT type of CTCs [20]. CTCs captured by CSV antibody expressed other EMT markers as well, such as Snail, Slug and Twist, suggesting that CSV could be used as a marker to isolate EMT type of CTCs [20]. Uses of CSV antibody to capture mesenchymal CTCs have been validated in breast, prostate, colorectal and pancreatic cancers [2124 ]. Mesenchymal CTCs are believed to have more clinical significance. The presence of mesenchymal CTCs is usually associated with disease progression and worse prognosis [25]. In a comparative study between early and metastatic patients of $\mathrm{BCa}$, mesenchymal CTCs were identified in $73 \%$ and $100 \%$ of patients [26]. Furthermore, expression of mesenchymal markers correlates with lymph node involvement. Taken together, it, suggests that the EMT phenotype is directly related to the metastatic potential of CTCs [27]. 
104

105

106

107

108

109

110

111

112

113

114

115

116

117

118

119

120

121

122

intermediate phenotype [17-18]. Tan et al showed that every cancer has an unique cancer-specific

EMT signature and EMT spectrums varied between different cell lines and tumors [28]. EpCAM

and CSV can be used as targets to isolate the epithelial and mesenchymal subtypes of CTCs,

respectively. Studies in breast and pancreatic cancers have shown the use of CSV is more efficient

to capture CTCs than EpCAM $[21,24]$. This study aimed at defining a suitable CTC enrichment

antibody for each solid tumor by comparing the CTC detection rates with EpCAM or CSV antibodies in different solid tumors.

\section{Methods and Materials}

\section{Patients}

detection between April 2017 and May 2018 in Zhejiang University Medical College Affiliated

Sir Run Run Shaw Hospital, Zhejiang University Medical College Second Affiliated Hospital and

Zhejiang Cancer Hospital were included in the study. Inclusion criteria for cancer patients were as

follows: (1) patients were diagnosed with confirmed cancer; (2) patients were treatment-naive

before CTC detection; (3) patients had negative history of other malignancy within five years prior

CTC detection. Patients of the following descriptions were excluded and rejected from the study:

(1) patients were pregnant or breast-feeding at time of CTC detection or surgery; (2) patients with more than one type of cancer; (3) patients had other conditions which investigators thought was 
123 not suitable for the study. Healthy people were defined as people without any clinical symptoms 124 of any disease at time time of CTC detection. In total, 72 healthy people and 690 cancer patients 125 were recruited, comprising 140 LC, 85 CRC, 92 BCa, 70 gastric cancer (GC), 66 pancreatic ductal 126 adenocarcinoma (PDAC), 50 cervical cancer (CC), 54 with head and neck squamous cell 127 carcinoma (HNSCC), 31 EC, 21 brain cancer (BC), 19 HCC, 25 prostate cancer (PCa), 14 ovarian 128 cancer (OC), 13 bladder cancer (UC), and 10 sarcoma. Detailed participant information was 129 summarized in the supplement Table 1. This study followed the principles established in the 130 Declaration of Helsinki and was approved by the ethics committee of each participated hospitals 131 with IRB number, Qi Xie Lin Chuang Shi Yan 20180427-1, （2018）Lun Shen Shi Ji Di（ 013 ) 132 Hao, and 2015-01-45 Hao, respectively.

\section{CTC detection}

A total of 853 CTC results from 690 cancer patients and 72 healthy people were collected.

91 cancer patients had CTC detection for both epithelial and mesenchymal CTCs at same time.

137 CTCs were detected by CytoSorter ${ }^{\circledR}$ (Hangzhou Watson Biotech, Hangzhou, China), using 138 CytoSorter ${ }^{\circledR}$ circulating epithelial cells detection kit (EpCAM) and/or mesenchymal cells detection 139 kit (CSV). Biotin-labeled CTC capturing antibodies were anti-EpCAM monoclonal antibody 140 (Biotin) (DH0023, Abnova, Taiwan) and cell-surface vimentin (CSV) monoclonal antibody, clone

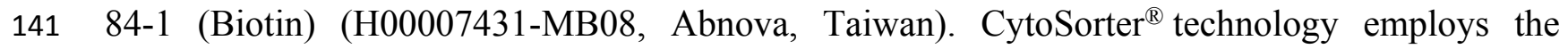
142 positive selection for CTCs by utilizing a streptavidin nanoarray on a Chip, CytoChipNano, which 
143 can be then coated with the biotin-labeled capturing antibody, and immunofluorescence staining,

144 in order to isolate and identify CTCs. CytoSorter ${ }^{\circledR}$ can be used with any biotin-labeled antibody to 145 capture desired targeted CTCs. EpCAM and CSV antibodies were coated on the chips separately 146 to enrich the epithelial and mesenchymal types of CTCs, respectively. Epithelial CTCs are 147 identified as PanCK-positive, CD45-negative and DAPI-positive cells, while mesenchymal CTCs 148 are identified as CSV-positive, CD45-negative and DAPI-positive cells. CTC detection procedure 149 was following the manufacturer protocol and was described in the previous study [24, 29]. An 150 epithelial CTC was identified as a PanCK-FITC+, CD45-PE-, and DAPI+ cell, while a 151 mesenchymal CTC was identified as a CSV-FITC+, CD45-PE-, and DAPI+ cell.

\section{Statistical analysis} two-sided $\mathrm{p}$ value less than 0.05 was considered statistically significant. 

different solid tumors and 23 healthy people, were included for analysis. An epithelial CTC is

164

165

166

167

168

169

170

171

172

173

174

175

176

177

178

179

180

181

shown in Figure 1A as indicated by the yellow arrow. CTCs were detected in 197 out of 318 cancer

patients, and 2 out of 23 healthy people as shown in Table 1. Healthy people usually have CTCs no more than 2, while cancer patients have usually CTCs more than 1. Epithelial CTCs detected by CytoSorter ${ }^{\circledR}$ might be used as a diagnostic aid for cancer screening with a sensitivity and specificity of 0.619 and 0.913 , respectively. Average CTC detection rate with EpCAM is 61.95\%. No epithelial CTC was found in sarcoma patients, which is in accordance with the expectation since sarcoma is a mesenchymal type of tumors. As shown in Figure 2A, EpCAM has the highest CTC detection rate of $84.09 \%$ in $\mathrm{CRC}$, followed by $\mathrm{BCa}(78.32 \%)$. EpCAM has CTC detection rates less than $50 \%$ in $\mathrm{HCC}(25 \%)$, PDAC (32.5\%) and OC (33.33\%). The CTC detection rate is not associated with patients' gender or age (both $\mathrm{P}>0.05)$.

\section{Identification of mesenchymal CTCs in different solid tumors}

512 CTC results for mesenchymal CTCs, including 463 cancer patients with 14 different solid tumors and 49 healthy people, we collected for analysis. A mesenchymal CTC is shown in Figure 1B as indicated by the yellow arrow. Mesenchymal CTCs were detected in 325 out of 463 cancer patients, and in 5 out of 49 healthy people as shown in Table 1. Like the EpCAM-based kit, healthy people have a maximum of 1 CTC detected, while cancer patients have usually more than $1 \mathrm{CTCs.}$ Thus, it indicates as well that mesenchymal CTCs captured by CSV antibody might be used as a screening aid for cancers with a sensitivity and specificity of 0.702 and 0.898 , respectively.. CSV 
182 has the highest CTC detection rate of $90 \%$ in the mesenchymal cancer, sarcoma. Beside sarcoma,

$183 \mathrm{CSV}$ has the highest CTC detection rate of $85.71 \%$ in $\mathrm{BC}$, followed by UC $(84.62 \%), \mathrm{OC}(83.33 \%)$

184 and $\mathrm{BCa}(81.82 \%)$. As shown in Figure 2B, CTC detection rates with CSV in all 14 solid tumors

185 are all over $60 \%$, with an average detection rate of $70.19 \%$. The CTC detection rate is not

186 associated with patients' gender or age (both $\mathrm{P}>0.05)$.

Comparison of CTC detection rates with EpCAM or CSV antibody in 12 different solid tumors

As shown in Table 1 and Figure 2C, CSV has better performances than EpCAM in most

cancers in regarding CTC detection rates, except for CRC. In CRC, EpCAM has a higher CTC

detection rate of $84.09 \%$ than CSV (67.35\%). CSV shows much better performances of $67.39 \%$,

$73.68 \%$ and $83.33 \%$, than EpCAM (32.5\%, 25\% and 33.33\%, respectively) in PDAC, HCC and

OC. In LC and EC, CSV performs slightly better than EpCAM (77.55\% VS $61.29 \%$ and 71.43\%

VS 57.14\%, respectively. In BCa, GC, HNSCC, CC and PCa, CSV and EpCAM have similar performances.

Comparison of CTC enumerations with EpCAM and CSV antibodies from the same blood drawings in the same patients at same time, including $20 \mathrm{LC}, 8 \mathrm{CRC}, 20 \mathrm{PDAC}, 10 \mathrm{GC}, 10 \mathrm{BCa}, 4 \mathrm{HCC}, 8 \mathrm{CC}, 4 \mathrm{EC}, 4 \mathrm{OC}$ and are higher than those with EpCAM. Although there is no statistical significance, patients with more 
201

202

203

204

205

206

207

208

209

210

211

212

213

214

215

216

217

epithelial CTCs tend to have more mesenchymal CTCs. CSV is more efficient to capture CTCs in PDAC patients than EpCAM as shown in Table $2(\mathrm{P}=0.0011)$. As for other cancers, no correlation is found between CTC enumerations by EpCAM and CSV.

\section{Discussions}

different solid tumors. Especially for EpCAM, the rates vary a lot from $84.09 \%$ in CRC to $25 \%$ in HCC. CTC detection rates with EpCAM antibody are quite in accordance with EpCAM expression profiles in different cancers. According to the Cancer Genome Atlas (TCGA) dataset, EpCAM is expressed in the cytoplasm and cell membranes of glandular cells, and most abundant in the gastrointestinal tract (colon, rectum and gallbladder) and thyroid gland as shown in supplement Figure 2 [30]. Furthermore, CRC has the lowest EMT score among all cancers [28]. Taken together, the high expression of EpCAM in colon and rectum and low EMT in CRC might explain why EpCAM has the highest CTC detection rate in CRC [28, 30-31]. On the contrary, there is a very low expression of EpCAM in the ovary and liver [30-31]. Therefore, EpCAM have lower CTC detection rates of $33.33 \%$ and $25 \%$ in OC and HCC, respectively. EpCAM is moderately expressed in lung, nasopharynx, breast, cervix, male organs and pancreas [30], and thus CTC detection rates are ranged mostly from $50 \%$ to $70 \%$ in these relevant solid tumors. Studies have shown that more mesenchymal CTCs would be found in patients at advanced cancer stage [26] and patients with more mesenchymal CTCs have higher metastasis potentials [18]. Although 
221 EpCAM is moderately expressed in pancreas, the CTC detection rate is only $32.5 \%$. It may be due

222 to that most PDAC patients enrolled in this study were already at advanced cancer stage at time of 223 diagnosis.

Due to the vimentin's unique characteristics of translocating from intracellular region to the cell surface in cells undergoing EMT [32], CSV has been proposed to be the target for the isolation of mesenchymal CTCs [20]. Our results show that the use of CSV antibody to detect CTCs would generate higher CTC detection rates in most solid tumors compared to the EpCAM, which is consistent with previous findings in $\mathrm{BCa}$ and PDAC $[21,24]$. Furthermore, CTC detection rates with CSV are all over $60 \%$ in general. Regarding the CTC detection rates, CSV performs much better than EpCAM in PDAC, HCC and OC. As reported by the TCGA dataset, HCC has low, while OC has high EpCAM expression [30]. However, both have high EMT scores [28], indicating most cancer cells from $\mathrm{HCC}$ and $\mathrm{OC}$ would undergo EMT, which might explain why CSV antibody performs better in these two cancers.

GC has a low EMT score and hematogenous metastasis is a rare phenomenon in early stage of GC, 
by the limited sample sizes or that PDAC patients recruited were at advanced cancer stage while patients of other cancers were at mixed cancer stages.. virus infection or fibrosis lesion [33-35]. Vimentin is normally expressed in mesenchymal cells, such as white blood cells (WBC), as a component of cytoskeleton [19]. A damaged WBC might also lead to false-positive results. and ensure tumor cells' survival in the circulation [36], the role of mesenchymal CTCs in metastasis remains under debate [37-38]. Some believe that EMT is of great importance in the 
260 suggested that EMT is not required for metastasis [37-38]. A recent in vivo animal study 261 demonstrated that epithelial CTCs with restricted mesenchymal transition are a major source of 262 metastases in BCa [39]. Still more studies support that mesenchymal CTCs are more important to 263 predict patients' survival outcomes [17, 40-41]. But evidences suggest that poor prognosis of 264 patients with more mesenchymal CTCs might be due to the increased chemoresistance rather than metastasis potential [42-43]. The EMT spectrums differ from tumors to tumors [28]. A low EMT score does not indicate a restricted metastasis potential. Although CRC has the lowest EMT score, 267 it can still easily develop metastasis. Each cancer may use different strategies to proceed 268 metastasis. capturing antibodies tested. Comparison of CTC detection rates with different CTC capturing antibodies should be performed in the same subjects to avoid the personal interference. Since it is a retrospective study, we were only able to collect 91 subjects who had CTC detection with both EpCAM and CSV antibodies. Only LC, BCa, and GC have sample sizes more than 50, and no patients with benign tumors were included for the analyses. Due to the small sample size, no correlation of CTCs with patients' pathological characteristics is found in most solid tumor. As 
280

281

282

283

284

285

286

287

288

289

290

291

292

293

294

295

296

297

298

299

antigen, such as human epidermal growth factor receptor 2 (HER2) for BCa and prostate specific membrane antigen (PSMA) for PCa, should be also included in this study. CTCs are heterogeneous, therefore, no single CTC capturing antibody is able to isolate all CTCs. Combination of multiple capturing antibodies should increase the CTC detection efficiency. It is reported that combined antibodies against folic acid (FA), epidermal growth factor receptor (EGFR) and vimentin can increase the CTC detection rate in LC [44]. Thus, combination of multiple CTC capturing antibodies should be also included in this study.

Our results show that EpCAM and CSV antibodies have different CTC detection rates across different tumors. Both EpCAM and CSV are not perfect markers for the isolation of CTCs. EpCAM is not expressed in every epithelial tumor [30] and may be lost in cells undergoing EMT [16]. CSV is expressed in EMT cells, apoptotic neutrophils, virus-infected cells and myofibroblasts $[19,33-35]$. The use of CSV to detect CTCs in the blood may generate some false-positive results. Since there is currently no other better markers, both are acceptable to detect CTCs in certain tumors. Based on our results, EpCAM can be used to isolate CTCs in EpCAM highly expressing tumors and in tumors with low EMT scores, such as CRC, LC, GC, BCa, EC, HNSCC, CC and PCa. CSV can be generally used to enrich CTCs in most solid tumors with acceptable CTC detection rates more than $60 \%$.

\section{Conclusions}

The results of this study showed that EpCAM can be used as a target to isolate CTCs in CRC, LC, GC, BCa, EC, HNSCC, CC and PCa, but not suitable in PDAC, HCC and OC, while CSV can 
300 be used in most solid tumors. However, these results should be prospectively validated with bigger

301 sample sizes.

302

303

\section{- Ethical approval}

304

305

306

307

308

309

310

311

312

313

314

315

316

317

318

319

320

321

This study followed the principles established in the Declaration of Helsinki and was approved by the ethics committee of Zhejiang University Medical College Affiliated Sir Run Run Shaw Hospital, Zhejiang University Medical College Second Affiliated Hospital and Zhejiang Cancer Hospital with IRB number, Qi Xie Lin Chuang Shi Yan 20180427-1, （2018 ) Lun Shen Shi Ji Di ( 013 ) Hao, and 2015-01-45 Hao, respectively.

\section{- Informed consent}

Written informed consent was obtained from each patient and healthy individual.

\section{References}

[1] Lozar T, Gersak K, Cemazar M, Kuhar CG, Jesenko T. The biology and clinical potential of circulating tumor cells. Radiol Oncol. 2019 May 8;53(2):131-147.

[2] Edge SB, Compton CC. The American Joint Committee on Cancer: the 7th edition of the AJCC cancer staging manual and the future of TNM. Ann Surg Oncol. 2010;17(6):1471-1474.

[3] Chinese expert consensus on screening and management of lung cancer. Guoji Huxi Zazhi. 2019;39(21):1604-1615.

[4] Chinese expert consensus on clinical applications of circulating tumor cells in colorectal cancer. J Laparoscopic Surg. 2019;24(001):74-80.

[5] 2019 Chinese Society of Clinical Oncology (CSCO) guidelines for breast cancer. Zhonghua Yi 
322

323

324

325

326

327

328

329

330

331

332

333

334

335

336

337

338

339

340

341

342

343

344

345

346

347

348

349

350

Xue Za Zhi. 2018;98(16):1213-1217.

[6] Chinese expert consensus on clinical applications of circulating tumor cells in esophageal squamous cell carcinoma. Shandong Med J. 2019;59(23):1-5.

[7] Chinese expert consensus on clinical applications of circulating tumor cells in hepatocellular carcinoma. Chinese J Laparoscopic Surg. 2019;12(4):193-198.

[8] Chinese medical association: Chinese expert consensus on early diagnosis and treatment of pancreatic cancer. Chinese J Onco. 2020;42(09):706-712.

[9] Butler TP, Gullino PM. Quantitation of cell shedding into efferent blood of mammary adenocarcinoma. Cancer Res. 1975 Mar;35(3):512-6.

[10] Tellez-Gabriel M, Heymann MF, Heymann D. Circulating Tumor Cells as a Tool for Assessing Tumor Heterogeneity. Theranostics. 2019 Jun 19;9(16):4580-4594.

[11] Trzpis M, McLaughlin PM, de Leij LM, Harmsen MC. Epithelial cell adhesion molecule: more than a carcinoma marker and adhesion molecule. Am J Pathol. 2007 Aug;171(2):386-95.

[12] https://www.cellsearchctc.com/

[13] Yang J, Weinberg RA. Epithelial-mesenchymal transition: at the crossroads of development and tumor metastasis. Dev Cell. 2008;14(6):818-829.

[14] Satelli A, Mitra A, Brownlee Z, Xia X, Bellister S, Overman MJ, Kopetz S, Ellis LM, Meng QH, Li S. Epithelial-mesenchymal transitioned circulating tumor cells capture for detecting tumor progression. Clin Cancer Res. 2015 Feb 15;21(4):899-906.

[15] Kalluri R. EMT: when epithelial cells decide to become mesenchymal-like cells. J Clin Invest. 2009 Jun;119(6):1417-9.

[16] Chaw SY, Abdul Majeed A, Dalley AJ, Chan A, Stein S, Farah CS. Epithelial to mesenchymal transition (EMT) biomarkers--E-cadherin, beta-catenin, APC and Vimentin--in oral squamous cell carcinogenesis and transformation. Oral Oncol. 2012 Oct;48(10):997-1006.

[17] Pastushenko I, Blanpain C. EMT Transition States during Tumor Progression and Metastasis. Trends Cell Biol. 2019 Mar;29(3):212-226.

[18] Yu M, Bardia A, Wittner BS, Stott SL, Smas ME, Ting DT, Isakoff SJ, Ciciliano JC, Wells MN, Shah AM, Concannon KF, Donaldson MC, Sequist LV, Brachtel E, Sgroi D, Baselga J, Ramaswamy S, Toner M, Haber DA, Maheswaran S. Circulating breast tumor cells exhibit 
351 dynamic changes in epithelial and mesenchymal composition. Science. 2013 Feb 352 1;339(6119):580-4.

353 [19] Satelli A, Li S. Vimentin in cancer and its potential as a molecular target for cancer therapy. 354 Cell Mol Life Sci. 2011 Sep;68(18):3033-46.

355 [20] Satelli A, Mitra A, Cutrera JJ, Devarie M, Xia X, Ingram DR, Dibra D, Somaiah N, Torres 356 KE, Ravi V, Ludwig JA, Kleinerman ES, Li S. Universal marker and detection tool for human 357 sarcoma circulating tumor cells. Cancer Res. 2014 Mar 15;74(6):1645-50.

358 [21] Satelli A, Brownlee Z, Mitra A, Meng QH, Li S. Circulating tumor cell enumeration with a 359 combination of epithelial cell adhesion molecule- and cell-surface vimentin-based methods for 360 monitoring breast cancer therapeutic response. Clin Chem. 2015 Jan;61(1):259-66.

361 [22] Satelli A, Batth I, Brownlee Z, Mitra A, Zhou S, Noh H, Rojas CR, Li H, Meng QH, Li S. 362 EMT circulating tumor cells detected by cell-surface vimentin are associated with prostate cancer 363 progression. Oncotarget. 2017 Jul 25;8(30):49329-49337.

364 [23] Satelli A, Batth IS, Brownlee Z, Rojas C, Meng QH, Kopetz S, Li S. Potential role of nuclear 365 PD-L1 expression in cell-surface vimentin positive circulating tumor cells as a prognostic marker 366 in cancer patients. Sci Rep. 2016 Jul 1;6:28910.

367 [24] Wei T, Zhang X, Zhang Q, Yang J, Chen Q, Wang J, Li X, Chen J, Ma T, Li G, Gao S, Lou 368 J, Que R, Wang Y, Dang X, Zheng L, Liang T, Bai X. Vimentin-positive circulating tumor cells 369 as a biomarker for diagnosis and treatment monitoring in patients with pancreatic cancer. Cancer 370 Lett. 2019 Jun 28;452:237-243.

371 [25] Jolly MK, Somarelli JA, Sheth M, Biddle A, Tripathi SC, Armstrong AJ, Hanash SM, Bapat 372 SA, Rangarajan A, Levine H. Hybrid epithelial/mesenchymal phenotypes promote metastasis and 373 therapy resistance across carcinomas. Pharmacol Ther. 2019 Feb;194:161-184.

374 [26] Kallergi G, Papadaki MA, Politaki E, Mavroudis D, Georgoulias V, Agelaki S. Epithelial to 375 mesenchymal transition markers expressed in circulating tumour cells of early and metastatic 376 breast cancer patients. Breast Cancer Res. 2011 Jun 10;13(3):R59.

377 [27] Markiewicz A, Ksi $\square \square$ kiewicz M, Seroczy $\square$ ska B, Skokowski J, Szade J, We $\square$ nicka$\mathrm{Ja} \square$ kiewicz M, Zaczek AJ. Heterogeneity of mesenchymal markers expression-molecular profiles 379 of cancer cells disseminated by lymphatic and hematogenous routes in breast cancer. Cancers 
380

381

382

383

384

385

386

387

388

389

390

391

392

393

394

395

396

397

398

399

400

401

402

403

404

405

406

407

408

(Basel). 2013 Nov 8;5(4):1485-503.

[28] Tan TZ, Miow QH, Miki Y, Noda T, Mori S, Huang RY, Thiery JP. Epithelial-mesenchymal transition spectrum quantification and its efficacy in deciphering survival and drug responses of cancer patients. EMBO Mol Med. 2014 Oct;6(10):1279-93.

[29] Zheng W, Zhang Y, Guo L, Wang S, Fang M, Mao W, Lou J. Evaluation of therapeutic efficacy with CytoSorter ${ }^{\circledR}$ circulating tumor cell-capture system in patients with locally advanced head and neck squamous cell carcinoma. Cancer Manag Res. 2019 Jun 27;11:5857-5869.

[30] https://www.proteinatlas.org/ENSG00000119888-EPCAM/tissue

[31] Spizzo G, Fong D, Wurm M, Ensinger C, Obrist P, Hofer C, Mazzoleni G, Gastl G, Went P. EpCAM expression in primary tumour tissues and metastases: an immunohistochemical analysis. J Clin Pathol. 2011 May;64(5):415-20.

[32] Mitra A, Satelli A, Xia X, Cutrera J, Mishra L, Li S. Cell-surface Vimentin: A mislocalized protein for isolating csVimentin(+) CD133(-) novel stem-like hepatocellular carcinoma cells expressing EMT markers. Int J Cancer. 2015 Jul 15;137(2):491-6.

[33] Moisan E, Girard D. Cell surface expression of intermediate filament proteins vimentin and lamin B1 in human neutrophil spontaneous apoptosis. J Leukoc Biol. 2006 Mar;79(3):489-98.

[34] Du N, Cong H, Tian H, Zhang H, Zhang W, Song L, Tien P. Cell surface vimentin is an attachment receptor for enterovirus 71. J Virol. 2014 May;88(10):5816-33.

[35] Song I, Ise H. Development of a Gene Delivery System of Oligonucleotides for Fibroses by Targeting Cell-Surface Vimentin-Expressing Cells with N-Acetylglucosamine-Bearing PolymerConjugated Polyethyleneimine. Polymers (Basel). 2020 Jul 7;12(7):1508.

[36] Jie XX, Zhang XY, Xu CJ. Epithelial-to-mesenchymal transition, circulating tumor cells and cancer metastasis: Mechanisms and clinical applications. Oncotarget. 2017 May 26;8(46):8155881571.

[37] Garber K. Epithelial-to-mesenchymal transition is important to metastasis, but questions remain. J Natl Cancer Inst. 2008 Feb 20;100(4):232-3, 239.

[38] Chui MH. Insights into cancer metastasis from a clinicopathologic perspective: EpithelialMesenchymal Transition is not a necessary step. Int J Cancer. 2013 Apr 1;132(7):1487-95.

[39] Liu X, Li J, Cadilha BL, Markota A, Voigt C, Huang Z, Lin PP, Wang DD, Dai J, Kranz G, 
409 Krandick A, Libl D, Zitzelsberger H, Zagorski I, Braselmann H, Pan M, Zhu S, Huang Y,

410 Niedermeyer S, Reichel CA, Uhl B, Briukhovetska D, Suárez J, Kobold S, Gires O, Wang H.

411 Epithelial-type systemic breast carcinoma cells with a restricted mesenchymal transition are a 412 major source of metastasis. Sci Adv. 2019 Jun 19;5(6):eaav4275.

413 [40] Hou J, Guo C, Lyu G. Clinical significance of Epithelial-Mesenchymal Transition typing of 414 circulating tumor cells in colorectal cancer. Colorectal Dis. 2019 Dec 23.

415 [41] Yang YJ, Kong YY, Li GX, Wang Y, Ye DW, Dai B. Phenotypes of circulating tumour cells 416 predict time to castration resistance in metastatic castration-sensitive prostate cancer. BJU Int. 4172019 Aug;124(2):258-267.

418 [42] Fischer KR, Durrans A, Lee S, Sheng J, Li F, Wong ST, Choi H, El Rayes T, Ryu S, Troeger 419 J, Schwabe RF, Vahdat LT, Altorki NK, Mittal V, Gao D. Epithelial-to-mesenchymal transition is 420 not required for lung metastasis but contributes to chemoresistance. Nature. 2015 Nov $42126 ; 527(7579): 472-6$.

422 [43] Zheng X, Carstens JL, Kim J, Scheible M, Kaye J, Sugimoto H, Wu CC, LeBleu VS, Kalluri 423 R. Epithelial-to-mesenchymal transition is dispensable for metastasis but induces chemoresistance 424 in pancreatic cancer. Nature. 2015 Nov 26;527(7579):525-530.

425 [44] Li G, Wang Y, Tan G, Liu Y, Xu Z, Feng H, Xing W, Xu Z. [Preliminary Study on Detection 426 of Circulating Tumor Cells in Lung Cancer by EGFR/Vimentin/Folic Acid Magnetic Sphere]. 427 Zhongguo Fei Ai Za Zhi. 2020 May 20;23(5):351-359. 


\section{Figure 1}

Immunofluorescent staining of CTCs.

Immunofluorescent staining of CTCs. (A) An epithelial CTC is identified as a DAPI (blue) positive, PanCK (FITC, green) positive and CD45 (PE, orange) negative cell as indicated by the yellow arrow. (B) A mesenchymal CTC is identified as a DAPI (blue) positive, CSV (FITC, green) positive and CD45 (PE, orange) negative cell as indicated by the yellow arrow. White blood cells, marked by white arrows, are identified as DAPI (blue) positive, FITC (green) negative and CD45 (PE, orange) positive cells. Scale bar represents $10 \mu \mathrm{m}$, immunofluorescent staining, X 20.

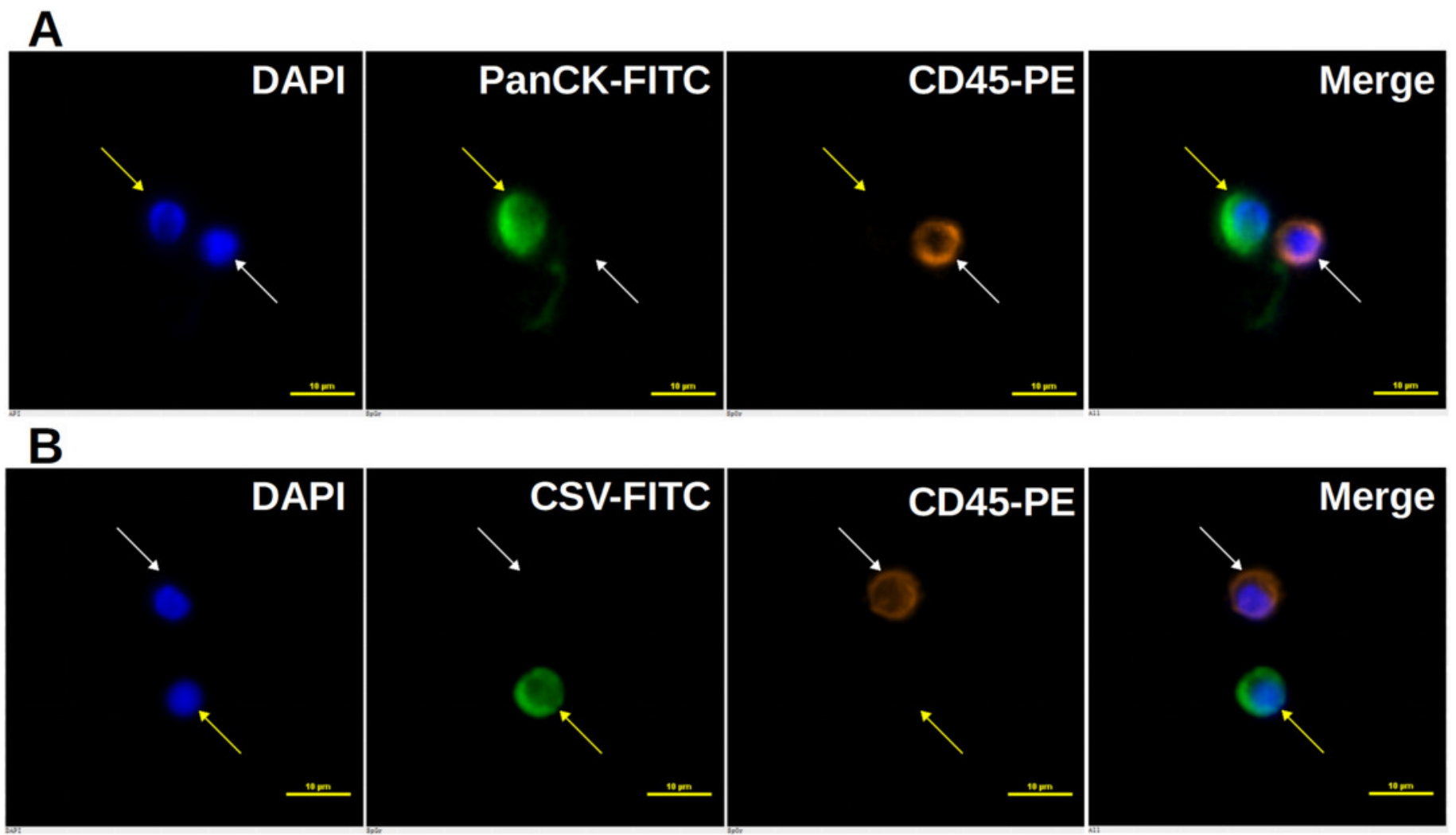




\section{Figure 2}

CTC detection rates in different solid tumors.

CTC detection rates in different solid tumors with EpCAM antibody as shown in (A), or with CSV antibody as shown in (B). Red dots represent the CTC detection rates and black bars represent the sample size. (C) Comparison of CTC detection rate with EpCAM and CSV antibodies in different solid tumors. $\mathrm{LC}=$ lung cancer; $\mathrm{CRC}=$ colorectal cancer; $\mathrm{BCa}=$ breast cancer; $\mathrm{GC}=$ gastric cancer; $\mathrm{PDAC}=$ pancreatic ductal adenocarcinoma; $\mathrm{CC}=$ cervical cancer; HNSCC = head and neck squamous cell carcinoma; $\mathrm{EC}=$ esophageal cancer; $\mathrm{BC}=$ brain cancer; $\mathrm{HCC}=$ hepatocellular carcinoma; $\mathrm{PCa}=$ prostate cancer; $\mathrm{OC}=$ ovarian cancer; $\mathrm{UC}=$ bladder cancer.
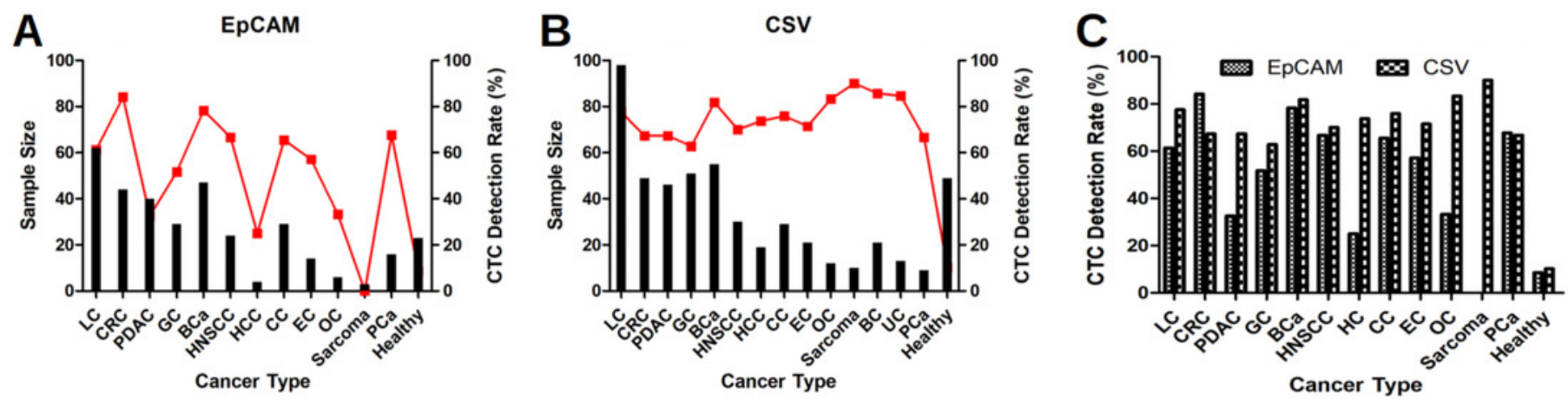


\section{Table 1 (on next page)}

CTC detection rates in different solid cancers with EpCAM or CSV antibody.

CTC detection rates in different solid cancers with EpCAM or CSV antibody. 
1 Table 1. CTC detection rates in different solid cancers with EpCAM or CSV antibody.

\begin{tabular}{|c|c|c|c|c|c|c|c|c|}
\hline \multirow{2}{*}{$\begin{array}{l}\text { Cancer } \\
\text { Type }\end{array}$} & \multirow[b]{2}{*}{$\mathrm{n}$} & \multicolumn{3}{|c|}{ EpCAM } & \multicolumn{4}{|c|}{ CSV } \\
\hline & & $\begin{array}{c}\text { CTC } \\
\text { Detected }\end{array}$ & $\begin{array}{l}\text { Detection } \\
\text { Rate }(\%)\end{array}$ & $\begin{array}{c}\text { CTCs Range } \\
\text { (/4 mL) }\end{array}$ & $\mathrm{n}$ & $\begin{array}{c}\text { CTC } \\
\text { Detected }\end{array}$ & $\begin{array}{l}\text { Detection } \\
\text { Rate (\%) }\end{array}$ & $\begin{array}{c}\text { CTCs Range } \\
(/ 4 \mathrm{~mL})\end{array}$ \\
\hline $\mathrm{LC}$ & 62 & 38 & 61.29 & $0-24$ & 98 & 76 & 77.55 & $0-22$ \\
\hline $\mathrm{CRC}$ & 44 & 37 & 84.09 & $0-20$ & 49 & 33 & 67.35 & $0-9$ \\
\hline PDAC & 40 & 13 & 32.50 & $0-3$ & 46 & 31 & 67.39 & $0-18$ \\
\hline $\mathrm{GC}$ & 29 & 15 & 51.72 & $0-13$ & 51 & 32 & 62.75 & $0-8$ \\
\hline $\mathrm{BCa}$ & 47 & 38 & 78.32 & $0-15$ & 55 & 45 & 81.82 & $0-8$ \\
\hline HNSCC & 24 & 16 & 66.67 & $0-36$ & 30 & 21 & 70.00 & $0-12$ \\
\hline $\mathrm{HCC}$ & 4 & 1 & 25.00 & $0-5$ & 19 & 14 & 73.68 & $0-14$ \\
\hline $\mathrm{CC}$ & 29 & 19 & 65.52 & $0-8$ & 29 & 22 & 75.86 & $0-7$ \\
\hline $\mathrm{EC}$ & 14 & 8 & 57.14 & $0-8$ & 21 & 15 & 71.43 & $0-7$ \\
\hline $\mathrm{OC}$ & 6 & 2 & 33.33 & $0-3$ & 12 & 10 & 83.33 & $0-7$ \\
\hline Sarcoma & 3 & 0 & 0.00 & 0 & 10 & 9 & 90.00 & $0-9$ \\
\hline $\mathrm{BC}$ & 0 & $\mathrm{~N} / \mathrm{A}$ & $\mathrm{N} / \mathrm{A}$ & $\mathrm{N} / \mathrm{A}$ & 21 & 18 & 85.71 & $0-6$ \\
\hline $\mathrm{UC}$ & 0 & $\mathrm{~N} / \mathrm{A}$ & $\mathrm{N} / \mathrm{A}$ & $\mathrm{N} / \mathrm{A}$ & 13 & 11 & 84.62 & $0-3$ \\
\hline $\mathrm{PCa}$ & 16 & 10 & 67.74 & $0-8$ & 9 & 6 & 66.67 & $0-11$ \\
\hline $\begin{array}{l}\text { Healthy } \\
\text { Control }\end{array}$ & 23 & 2 & 8.51 & $0-1$ & 49 & 5 & 10.20 & $0-1$ \\
\hline
\end{tabular}

2 EpCAM = epithelial cell adhesion molecule; $\mathrm{CSV}=$ cell surface vimentin; $\mathrm{n}=$ sample number;

$3 \mathrm{CTC}=$ circulating tumor cell $; \mathrm{LC}=$ lung cancer; $\mathrm{CRC}=$ colorectal cancer; $\mathrm{BCa}=$ breast cancer;

$4 \mathrm{GC}=$ gastric cancer; PDAC $=$ pancreatic ductal adenocarcinoma; $\mathrm{CC}=$ cervical cancer; $\mathrm{HNSCC}$ $5=$ head and neck squamous cell carcinoma; $\mathrm{EC}=$ esophageal cancer; $\mathrm{BC}=$ brain cancer; N/A = 
6 not available; $\mathrm{HCC}=$ hepatocellular carcinoma; $\mathrm{PCa}=$ prostate cancer; $\mathrm{OC}=$ ovarian cancer; $\mathrm{UC}$ 7 = bladder cancer.

8

9 


\section{Table 2 (on next page)}

Comparison of CTC enumerations with EpCAM and CSV antibodies in the same patients

Comparison of CTC enumerations with EpCAM and CSV antibodies in the same patients 
1 Table 2. Comparison of CTC enumerations with EpCAM and CSV antibodies in the same 2 patients

\begin{tabular}{|c|c|c|c|c|c|c|c|c|c|c|c|}
\hline \multirow{2}{*}{$\begin{array}{c}\text { Patient } \\
\text { No. }\end{array}$} & \multicolumn{2}{|c|}{ CTC Count } & \multirow{2}{*}{ P Value } & \multirow{2}{*}{$\begin{array}{c}\text { Patient } \\
\text { No. }\end{array}$} & \multicolumn{2}{|c|}{ CTC Count } & \multirow{2}{*}{ P Value } & \multirow{2}{*}{$\begin{array}{c}\text { Patient } \\
\text { No. }\end{array}$} & \multicolumn{2}{|c|}{ CTC Count } & \multirow{2}{*}{ P Value } \\
\hline & ЕpCAM & CSV & & & EpCAM & CSV & & & ЕpCAM & CSV & \\
\hline \multicolumn{3}{|c|}{$L C(n=20)$} & & \multicolumn{3}{|c|}{$P D A C(n=20)$} & & \multicolumn{3}{|c|}{$B C a(n=10)$} & \\
\hline 1 & 0 & 3 & & 29 & 0 & 2 & & 59 & 6 & 10 & \\
\hline 2 & 0 & 0 & & 30 & 1 & 4 & & 60 & 5 & 6 & \\
\hline 3 & 0 & 5 & & 31 & 0 & 3 & & 61 & 0 & 1 & \\
\hline 4 & 0 & 4 & & 32 & 0 & 3 & & 62 & 9 & 6 & \\
\hline 5 & 0 & 3 & & 33 & 1 & 0 & & 63 & 0 & 3 & \\
\hline 6 & 5 & 5 & & 34 & 0 & 7 & & 64 & 4 & 0 & 0.9051 \\
\hline 7 & 1 & 2 & & 35 & 3 & 11 & & 65 & 3 & 1 & \\
\hline 8 & 4 & 2 & & 36 & 1 & 0 & & 66 & 0 & 1 & \\
\hline 9 & 5 & 3 & & 37 & 3 & 11 & & 67 & 3 & 1 & \\
\hline & & & 0.1283 & & & & 0.0011 & & & & \\
\hline 10 & 7 & 11 & & 38 & 0 & 4 & & 68 & 0 & 0 & \\
\hline 11 & 24 & 16 & & 39 & 0 & 7 & & $H C C(n$ & $=4)$ & & \\
\hline 12 & 12 & 13 & & 40 & 0 & 1 & & 69 & 0 & 0 & \\
\hline 13 & 8 & 9 & & 41 & 0 & 8 & & 70 & 0 & 2 & \\
\hline 14 & 18 & 3 & & 42 & 0 & 2 & & 71 & 0 & 1 & \\
\hline 15 & 2 & 4 & & 43 & 2 & 1 & & 72 & 5 & 4 & \\
\hline 16 & 18 & 22 & & 44 & 0 & 17 & & $C C(n=$ & & & \\
\hline 17 & 20 & 22 & & 45 & 0 & 7 & & 73 & 0 & 1 & \\
\hline 18 & 11 & 14 & & 46 & 1 & 4 & & 74 & 9 & 2 & \\
\hline
\end{tabular}




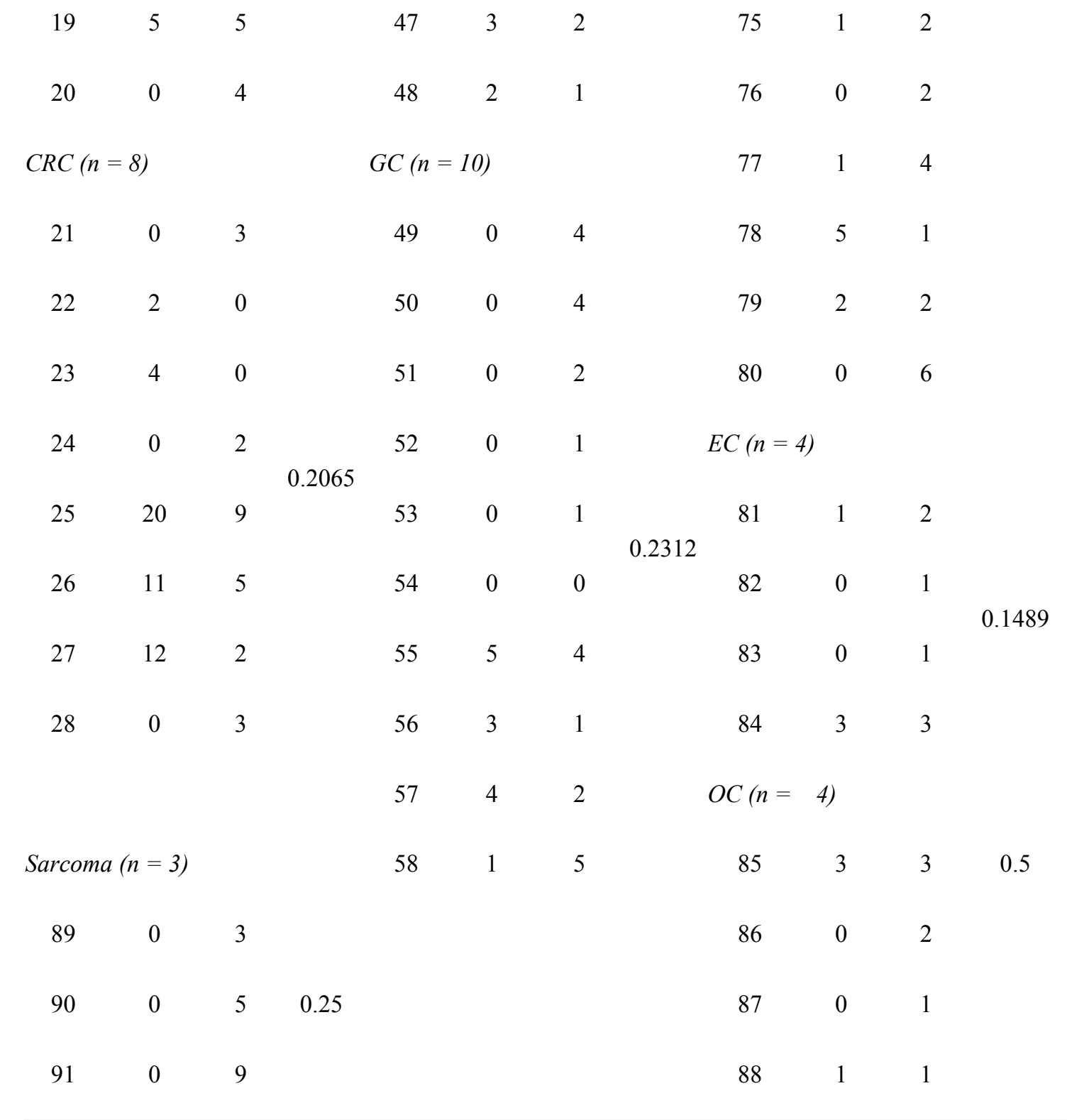

$3 \mathrm{CTC}=$ circulating tumor cell; EpCAM = epithelial cell adhesion molecule; $\mathrm{CSV}=$ cell surface vimentin; 4 No = number; $\mathrm{n}=$ sample number; $\mathrm{LC}=$ lung cancer; $\mathrm{CRC}=$ colorectal cancer; $\mathrm{BCa}=$ breast cancer; $\mathrm{GC}$ 5 = gastric cancer; $\mathrm{PDAC}=$ pancreatic ductal adenocarcinoma; $\mathrm{CC}=$ cervical cancer; $\mathrm{HNSCC}=$ head and 6 neck squamous cell carcinoma; $\mathrm{EC}=$ esophageal cancer; $\mathrm{BC}=$ brain cancer; $\mathrm{N} / \mathrm{A}=$ not available; $\mathrm{HCC}=$ 7 hepatocellular carcinoma; $\mathrm{PCa}=$ prostate cancer; $\mathrm{OC}=$ ovarian cancer; $\mathrm{UC}=$ bladder cancer. 